\title{
Near-diffraction-limited coherent emission from large aperture antiguided vertical-cavity surface-emitting laser arrays
}

\author{
Ling Bao, ${ }^{\text {a) }}$ Nam-Heon Kim, and Luke J. Mawst \\ Reed Center for Photonics, Department of Electrical Computer Engineering, University \\ of Wisconsin-Madison, 1415 Engineering Drive, Madison, Wisconsin 53706-1691
}

\author{
N. N. Elkin, V. N. Troshchieva, D. V. Vysotsky, and A. P. Napartovich \\ State Science Center Troitsk Institute for Innovation and Fusion Research (TRINITI), 142190 Trinti Troitsk \\ Moscow Region, Russia
}

(Received 25 July 2003; accepted 20 November 2003)

\begin{abstract}
We demonstrate that in-phase mode operation with a near-diffraction-limited beam can be realized in large aperture (up to 100 elements) antiguided vertical-cavity surface-emitting laser (VCSEL) arrays. A selective etching process with two-step metalorganic chemical vapor deposition is used for fabrication of the antiguided VCSEL array structures. Modal discrimination is enhanced by intentionally choosing a GaAs cap thickness so as to introduce suitable loss to array interelement regions. Far field patterns indicate in-phase mode operation from both triangular and rectangular geometry antiguided VCSEL arrays, which is in good agreement with theory. (C) 2004 American Institute of Physics. [DOI: 10.1063/1.1640799]
\end{abstract}

Coupled two-dimensional vertical-cavity surfaceemitting arrays are promising as high power (more than 10 $\mathrm{mW}$ ) coherent light sources for many applications such as telecommunications, printing, data storage etc. In addition, their two-dimensional periodicity of the gain and index make them ideal sources with which to study the properties of active photonic lattices. ${ }^{1}$ In the past, much effort has been directed towards realizing coherent emission from phaselocked vertical-cavity surface-emitting laser (VCSEL) arrays. However, previous work has shown that phase-locked VCSEL arrays tend to lase in the highest-order, out-of-phase, transverse mode. Mode discrimination is also low among various transverse array modes, and generally results in a mixture of high-order modes reaching laser threshold. ${ }^{2}$ Continuous wave $(\mathrm{cw})$ operation of $10 \times 10 \mathrm{VCSEL}$ arrays that utilized a metal grid defined structure to insure single higherorder mode operation has been reported. ${ }^{3}$ Larger aperture $(\sim 138 \mu \mathrm{m} \times 138 \mu \mathrm{m})$ coherent $20 \times 20$ VCSEL arrays have been reported previously using photon pumping, and they exhibited a four-lobe, multimode, far-field pattern. ${ }^{4}$ This behavior is characteristic of evanescent wave coupling in weakly positive index guided or gain guided arrays. ${ }^{5}$ To make "in-phase-mode-like emission," external phase shifters are required, although the result is still a relatively broad beam that is indicative of multimode operation. ${ }^{6}$ Thus, stable, high power, diffraction-limited beam operation has not been realized.

Compared to weak evanescent coupling in positive index guided arrays, antiguided structures are attractive due to the strong leaky-wave coupling between each element in the array. What is more, modal discrimination is also enhanced by array mode dependent lateral radiation loss. Thus, by properly choosing the index step and width of the coupling region, the in-phase array mode is favored to lase. Moreover, antiguided arrays are less vulnerable to thermal lensing and

${ }^{a)}$ Electronic mail: lbao@cae.wisc.edu gain spatial hole burning (GSHB) because of the strong built-in index step, which leads to stable beam operation at high output power. ${ }^{7}$ In 1999 Serkland et al. reported the observation of in-phase mode emission from two leaky-wave coupled VCSELs. ${ }^{8}$ Later, in 2000, diffraction-limited emission from two-dimensional $(2 \mathrm{D})(4 \times 4)$ leaky-wave coupled VCSEL arrays ${ }^{9,10}$ was reported by our group. In this work, we demonstrate the coherent scaling properties of antiguided 2D VCSEL arrays that achieve in-phase mode operation with near-diffraction-limited beams from 100 element arrays.

In antiguided arrays, resonant leaky-wave coupling for the in-phase (out-of-phase) mode occurs when the array interelement spacing corresponds to an odd (even) integral number of half waves of leaky-wave radiation within the interelement region. ${ }^{11}$ In antiguided VCSEL arrays, we find that mode selection is primarily a consequence of two mechanisms: ${ }^{10}$ (1) lateral edge radiation loss and (2) interelement loss. Intentionally adding an absorbing layer [such as an InGaAs quantum well $(\mathrm{QW})]$ in the interelement regions of the array has been done previously to suppress nonresonant modes (as well as guided modes), ${ }^{9,10}$ since nonresonant/guided modes exhibit high optical field intensity in interelement regions. A simpler technique, used here, utilizes metal absorption loss by choosing an optimal thickness of $p$-GaAs cap thickness. This technique was reported previously to fabricate metal grid VCSEL arrays, which operate in an out-of-phase array mode. ${ }^{3}$ The $2 \mathrm{D}$ array is constructed so that the emitting element regions are terminated by $p$-GaAs/air, and the interelement regions are terminated by $p$-GaAs/Ti:Pt:Au. A simple one-dimensional (1D) transfer matrix method has been used to calculate the loss in both interelement (Ti) and element regions (air). Figure 1 shows that the calculated loss changes periodically according to the GaAs cap thickness. Different resonant points for Ti and air occur as a result of phase shifting in the metal. Within a certain GaAs cap thickness range, e.g., $t$ cap $=25-75 \mathrm{~nm}$, 


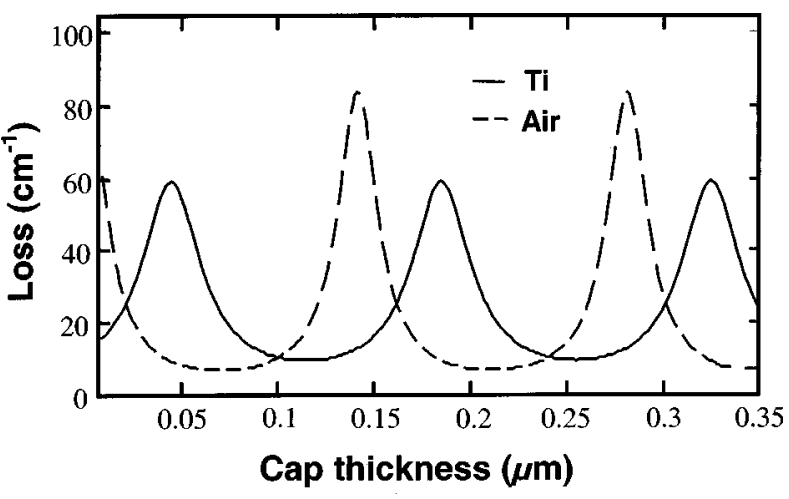

FIG. 1. Calculated loss vs GaAs cap layer thickness $(n-\mathrm{DBR}=32$ pairs; $p$ - $\mathrm{DBR}=23.5$ pairs).

higher loss can be introduced only to the interelement regions (Ti regions).

In our studies, the built-in index step and array pattern are achieved by a selective etching process and two-step metalorganic chemical vapor deposition growth. ${ }^{12}$ The element regions (6 $\mu \mathrm{m}$ wide with a $5 \mu \mathrm{m}$ wide metal opening) include 32 pairs of $\mathrm{AlAs} / \mathrm{GaAs} n$-distributed Bragg reflector (DBR), a 1- $\lambda$ optical cavity, which consists of three InGaAs quantum wells, GaAs barrier layers, and $\mathrm{Al}_{0.3} \mathrm{Ga}_{0.70} \mathrm{As}$ confinement layers for $980 \mathrm{~nm}$ emission, and 23.5 pairs of $\mathrm{Al}_{0.15} \mathrm{Ga}_{0.85} \mathrm{As} / \mathrm{Al} \mathrm{As} p$-DBRs. The interelement regions are formed by adding two thin spacer layers of GaAs and GaInP above the fourth pair of $\mathrm{Al}_{0.15} \mathrm{Ga}_{0.85} \mathrm{As} / \mathrm{AlAs} p$-DBR. According to effective index theory, for a VCSEL, ${ }^{13}$ a lateral effective index step $\Delta n$ for antiguiding forms due to redshifting of the optical resonance within the interelement regions. The interelement spacing, $s$, is varied so as to select either in-phase or out-of-phase array mode. Based on the 1D loss calculation, three different GaAs cap thicknesses, tcap in Fig. 1 have been chosen; 45, 57, and $70 \mathrm{~nm}$ (1/4 wave thickness). Both rectangular (four nearest neighbor) and triangular (six nearest neighbor) array configurations have been fabricated and studied.

Pulsed (100 ns, 1\% duty cycle) far-field measurements from both rectangular and triangular array configurations are performed. Near-diffraction-limited-beam, in-phase mode emission, in excellent agreement with the calculated far-field patterns, has been found in array structures as large as 10 $\times 10$ (100 elements) with $t$ cap $=0.045$ or $0.057 \mu \mathrm{m}$. Lasing occurs primarily in either the in-phase mode or out-of-phase mode, depending on the particular value of interelement spacing, although some devices have shown a mixture of both in-phase and out-of-phase mode or intermediate mode operation. In array structures with $t$ cap $=0.070 \mu \mathrm{m}$, most of the devices exhibit a complex mixture of in-phase, out-ofphase, intermediate mode. The measured far-field scans (horizontal and vertical) of a $10 \times 10$ rectangular array with $t$ cap $=0.045 \mu \mathrm{m}$ are shown in Fig. 2(b). The corresponding calculated far-field pattern is also shown in Fig. 2(a) for comparison. The measured center beam width is about $0.58^{\circ}$, which is close to the value estimated for the diffraction limit (DL) based on the total size of the aperture. Larger $20 \times 20$ array structures also operate primarily in phase, however, the beam width is three to four times the diffraction limit. The measured $P-I$ ( $1 \mu \mathrm{s}, 1 \%$ duty cycle) and corresponding farDownloaded 30 Nov 2006 to 128.104.198.120. Redistribution subje

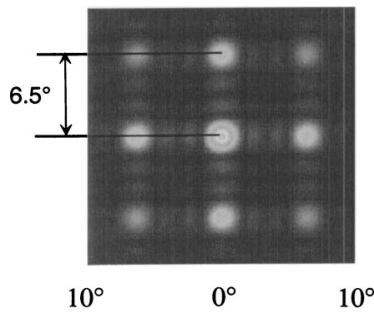

(a)

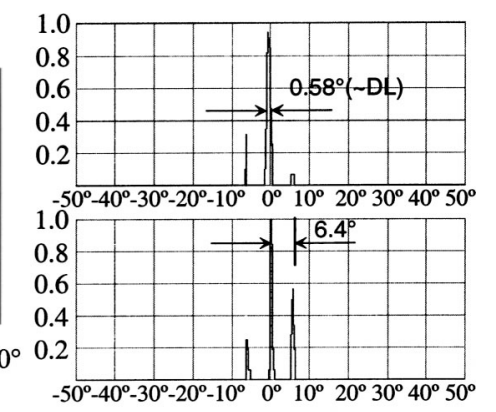

(b)

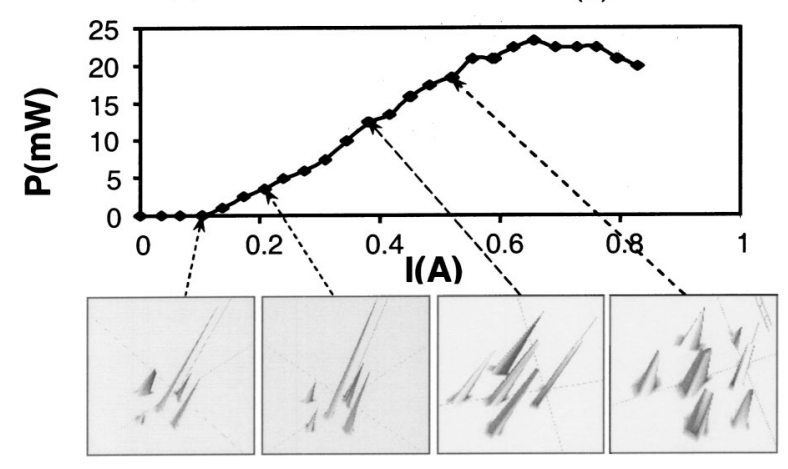

(c)
FIG. 2. (a) Calculated in-phase mode far field, (b) measured 1D cross section of far field (top: vertical, bottom: horizontal) in a $10 \times 10$ rectangular array (interelement width, $s=3.0 \mu \mathrm{m}$ ), (c) measured in-phase mode far field and power output vs the pulsed driving current (pulse width $=2 \mu \mathrm{s}$ ) in a $10 \times 10$ rectangular array $(s=3.0 \mu \mathrm{m})$

field interference patterns at various drive levels for the same device are shown in Fig. 2(c). From this, we can see that this device maintains a relatively stable far-field pattern with driving current up to $>5 \times I_{\text {th }}$, indicating that the in-phase mode dominates over a large current range. Nevertheless, broadening of the center lobe at high drive currents indicates the onset of additional (intermediate) array modes. The relatively low external differential efficiency $(\sim 3 \%)$ is believed to result from an unoptimized $p$-DBR pair number and large aperture array heating for the $1 \mu$ s pulse width utilized. To minimize heating, a junction-down configuration is preferred.

Triangular arrays have also been found to exhibit inphase mode operation. The measured far-field profile and corresponding calculated profile for a 19 element triangular array are shown separately in Figs. 3(b) and 3(a), respectively. The measured center beam width, $1.41^{\circ}$, is about 1.16 times the estimated diffraction limit based on the total aperture size. A three-dimensional (3D) view of the measured far-field pattern with driving current up to $200 \mathrm{~mA}$ (about $\left.4 \times I_{\text {th }}\right)$ is depicted in Fig. 3(c). We find the triangular arrays have an improved ability to maintain a stable in-phase mode pattern with an increase in drive current compared to rectangular arrays. We believe this is due to stronger element coupling in triangular arrays (more nearest neighbors), although detailed studies are still necessary. In addition, certain competing adjacent modes present in rectangular arrays, like the one with an even number of intensity peaks in one direction and an odd number of intensity peaks in the other direction, are no longer supported by the triangular array geometry.

The influence of interelement spacing on array modal behavior has also been studied. Devices which exhibit in-
to AIP license or copyright, see http://apl.aip.org/apl/copyright.jsp 


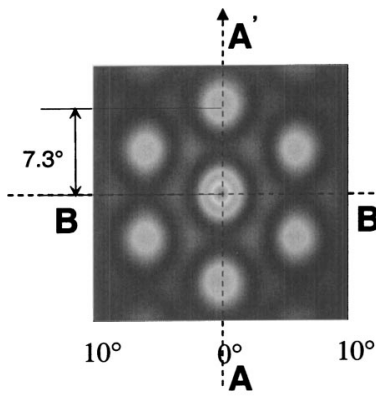

(a)

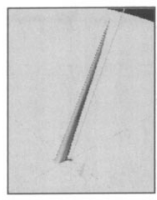

$\mathrm{I}=50 \mathrm{~mA}$

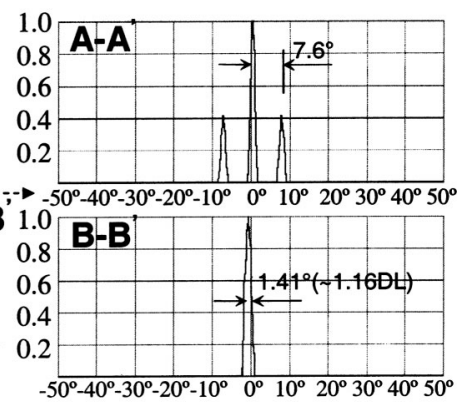

(b)

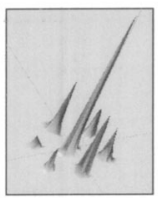

$\mathrm{I}=100 \mathrm{~mA}$

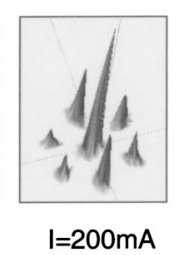

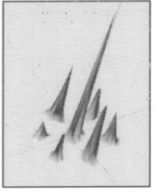

$I=150 \mathrm{~mA}$ ) (c)

FIG. 3. (a) Calculated in-phase mode far field, (b) measured 1D cross section of far field (top: vertical, bottom: horizontal) in a 19 element triangular array (interelement width, $s=2.0 \mu \mathrm{m}$ ), (c) measured in-phase mode far field vs the pulsed driving current (pulse width $=2100 \mathrm{~ns}$ ) in a 19 element triangular array (interelement width, $s=2.0 \mu \mathrm{m}$ )

phase mode and out-of-phase mode as a function of interelement spacing, $s$, are summarized in Fig. 4. For rectangular arrays, the in-phase mode is favored around $s=3.0 \mu \mathrm{m}$, whereas the out-of-phase mode is favored around $s$ $=1.5-2.0 \mu \mathrm{m}$. For triangular arrays, in-phase mode is favored around $s=3.5 \mu \mathrm{m}$ and out-of-phase mode is favored around $s=1.5 \mu \mathrm{m}$. The 1D lateral leaky-wave resonance condition can be calculated from the index step and interelement width $s .{ }^{10}$ From a 1D transfer matrix calculation, the index step is estimated to be $\Delta n=0.015$, resulting in a lateral leaky-wave wavelength of $2.98 \mu \mathrm{m}$. Therefore, the lateral resonance condition is satisfied for $s$ values around $1.5 \mu \mathrm{m}$ (for in-phase-mode) or $3 \mu \mathrm{m}$ (for out-of-phase mode). As expected from theory, the out-of-phase mode is generally favored at the in-phase mode resonance position, $s$ $\sim 1.5 \mu \mathrm{m}$, because of lower edge radiation loss for the nonresonant out-of-phase mode. Similarly, the in-phase mode is favored at the out-of-phase resonance position $s \sim 3.0 \mu \mathrm{m}$. Simulations studies using a 3D bidirectional beam propagation model ${ }^{12}$ are in good agreement with the measurements. These simulations also indicated that improved modal discrimination can be achieved by increasing the amount of loss in the interelement regions, thereby allowing the resonant mode to have the lowest threshold gain.

In conclusion, we have demonstrated near-diffractionlimited in-phase mode coherent emission from very large aperture (up to 100 elements) antiguided VCSEL arrays. Both rectangular and triangular antiguided VCSEL have demonstrated primarily in-phase mode operation with a relatively stable far-field pattern with an increase in drive current. Improved modal discrimination is expected to be real-

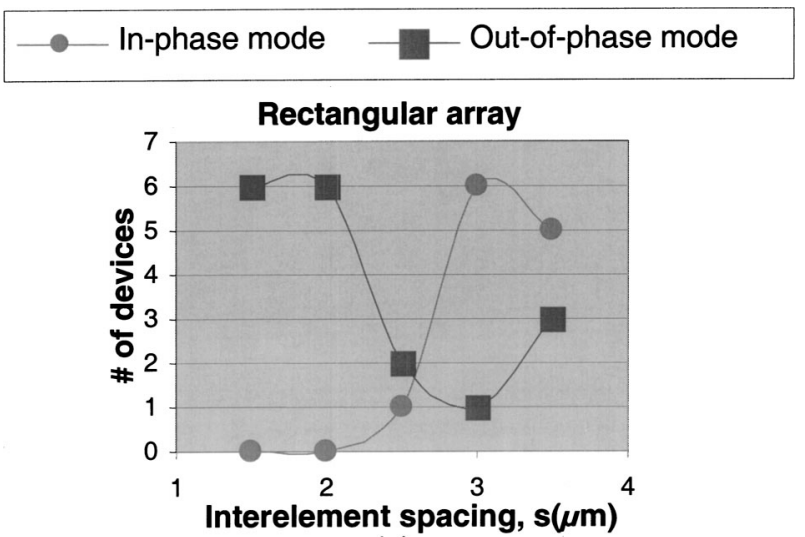

(a)

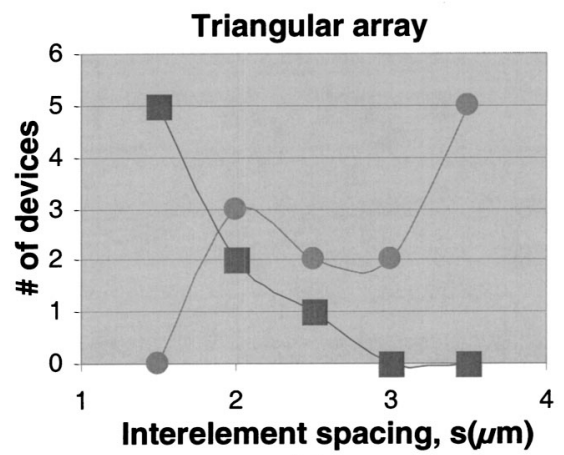

(b)

FIG. 4. Number of devices vs interelement spacing, $s(\mu \mathrm{m})$, for in-phase mode and out-of-phase mode. (a) Rectangular arrays. (b) Triangular arrays.

ized by incorporating increased interelement loss. Structure optimization and a suitable thermal package design are also needed to further increase output power in these large aperture array devices.

This work was supported by NSF Project No. 0139823. The authors at TRINITI also acknowledge partial support by Russian Foundation for Basic Research Project No. 02-0217101.

${ }^{1}$ L. J. Mawst, IEEE Circuits Devices Mag. 19, 34 (2003).

${ }^{2}$ Kuo-Liang Chen and Shyh Wang, Appl. Phys. Lett. 47, 555 (1985).

${ }^{3}$ F. Monti di Sopra, M. Brunner, H.-P. Gauggel, H. P. Zappe, M. Moser, R. Hover, and E. Kapon, Appl. Phys. Lett. 77, 2283 (2000).

${ }^{4}$ P. L. Gourley, M. E. Warren, G. R. Hadley, G. A. Vawter, T. M. Brennan, and B. E. Hammons, Appl. Phys. Lett. 58, 890 (1991).

${ }^{5}$ G. R. Hadley, Opt. Lett. 15, 1215 (1990).

${ }^{6}$ M. E. Warren, P. L. Gourley, G. A. Vawter, T. M. Brennam, B. E. Hammons, and K. L. Lear, Appl. Phys. Lett. 61, 1484 (1992).

${ }^{7}$ D. Botez, M. Jansen, L. J. Mawst, G. Peterson, and T. J. Roth, Appl. Phys. Lett. 58, 2070 (1991).

${ }^{8}$ D. K. Serkland, K. D. Choquette, G. R. Hadley, K. M. Geib, and A. A. Allerman, Appl. Phys. Lett. 75, 3754 (1999).

${ }^{9}$ D. Zhou and L. J. Mawst, Appl. Phys. Lett. 77, 2307 (2000).

${ }^{10}$ D. Zhou, L. J. Mawst, and Z. Dai, IEEE J. Quantum Electron. 38, 652 (2002).

${ }^{11}$ D. Botez, L. J. Mawst, G. L. Peterson, and T. J. Roth, IEEE J. Quantum Electron. 26, 482 (1990).

${ }^{12}$ N. N. Elkin, A. P. Napartovich, D. V. Vysotsky, V. N. Troshchieva, L. Bao, N. Kim, D. Zhou, and L. J. Mawst, Proc. SPIE 4994, 112 (2003).

${ }^{13}$ G. R. Hadley, Opt. Lett. 20, 1483 (1995). 